Aim(s)/objectives To ascertain if our service is following the 2014 BASHH recommendations for MSM STI screening.

Methods 97 MSM attending December 2014 to July 2015 coded T2 and T4, (Chlamydia and Gonorrhoea screening performed and Chlamydia, Gonorrhoea, HIV and Syphilis screening performed respectively), and MSM requesting post exposure prophylaxis (PEP) were included.

Results 3\% met Hepatitis C screening recommendations but were not offered testing. Hepatitis $\mathrm{C}$ risk factors were not always documented so it is likely more patients should have been offered Hepatitis C screening. Some tests were not indicated for every patient, for example Chlamydia and Gonorrhoea screening is not routinely offered until the 2 week visit for patients attending for PEP due to the 2 week window period for these infections.

Discussion/conclusion BASHH recommend 97\% of MSM attending a sexual health service with a new episode of care should be offered STI screening with $80 \%$ uptake. Targets for offering HIV and Syphilis screening were achieved but targets for offering Chlamydia, Gonorrhoea and Hepatitis B screening were not met and need to be improved upon. The results also highlighted screening for Hepatitis $\mathrm{C}$ risk factors (such as chemsex) needs to be routinely undertaken. The target for STI screening uptake in all areas was achieved. Repeat STI testing needs to be routinely offered to MSM.

\section{P195 DIAGNOSING RECENT HIV INFECTION IN AN URBAN SEXUAL HEALTH CENTRE: COULD MORE HAVE BE DONE TO PREVENT ACQUISITION?}

Eliza Walker*, Rebecca Gilson, Imali Fernando. NHS Lothian, Edinburgh, UK

\subsection{6/sextrans-2016-052718.244}

Background/introduction There are a number of strategies that may be employed by sexual health services to prevent HIV transmission, including motivational interviewing and Pre-Exposure Prophylaxis (PrEP). In order to utilise resources effectively, prevention strategies need to target those at high risk of acquiring HIV such as those having unprotected anal sex or who had known rectal infections.

Aim(s)/objectives We aimed to identify individuals in our cohort diagnosed with recently acquired HIV infection, review whether they had previously been accurately identified as high risk and what strategies had been employed to attempt reduce their risk.

Methods Electronic records of patients diagnosed HIV positive at an urban sexual health centre over a two year period were reviewed for timing of acquiring infection and previous engagement with sexual health services. Recently acquired infections were determined by: positive avidity, a negative test or a history of seroconversion symptoms in the 6-months prior to positive result.

Results 68 patients were diagnosed with HIV; 30 (44.1\%) were recently acquired infections. Of these, $13(43.3 \%)$ had attended a sexual health service in the year prior, 12 (92\%) of whom had been identified as at risk and had risks discussed by a healthcare professional.

Discussion/conclusion Almost half of our patients with recently acquired HIV had had contact with sexual health services in the year before their diagnosis, and the vast majority were identified at high risk. Being able to correctly identify patients at high risk of HIV has implications for using strategies such as PrEP in the future.
P197 EVALUATION OF THE CLINICAL UTILITY OF THE BECTON DICKINSON PROBETEC QX (BDQ) TRICHOMONAS VAGINALIS MOLECULAR DETECTION TEST IN TWO LARGE, URBAN GU MEDICINE SERVICES

${ }^{1}$ Craig Tipple, ${ }^{2}$ Michael Rayment* ${ }^{3}$ Monica Rebec, ${ }^{3}$ John Murray, ${ }^{2}$ Rachael Jones, 'Dawn Wilkinson. 'Department of GU Medicine, Jefferis Wing Centre for Sexual Health, St Mary's Hospital, Imperial College Healthcare NHS Trust, London, UK; ${ }^{2}$ Directorate of HIV and GU Medicine, West London Centre for Sexual Health, Chelsea and Westminster Hospital NHS Foundation Trust, London, UK; ${ }^{3}$ Department of Pathology, Charing Cross Hospital, Imperial College Healthcare NHS Trust, London, UK

\subsection{6/sextrans-2016-052718.245}

Background BASHH guidelines recommend molecular tests to aid diagnosis of Trichomonas vaginalis (TV), but many clinics use relatively insensitive techniques ( $\mathrm{pH}$, wet-prep microscopy (WPM) and culture).

Objectives To establish a laboratory pathway for TV testing with the BDQ assay, determine TV prevalence, and identify variables associated with TV detection.

Methods A prospective study of 900 women attending clinics for STI testing was undertaken. All were offered TV BDQ tests. Data collected: demographics, symptoms, results of near-patient tests and BDQ for TV, Chlamydia trachomatis (CT) and Neisseria gonorrhoeae (GC). Women with any positive TV result were treated and invited to attend for test of cure (TOC). Data were collected in Excel and analysed in SPSS.

Results 891 women had a TV BDQ test. 472 (53\%) were white, 143 (16\%) black; median age 28yrs. 499 (55\%) were symptomatic. Infections detected by BDQ: 11 TV (1.2\%), 3 GC (0.3\%) and 44 CT (4.9\%). Of BDQ+ TV infections: 8 (73\%) black, 7 (64\%) symptomatic, 4/7 (57\%) WPM+, 4/4 (100\%) pH > 4.5, 7/7 (100\%) Hay-Ison Grade 2, and 1/3 (33\%) TV culture+. Mean BDQ turn-around time: 3.44 days. All received treatment. 9/9 (100\%) were BDQ negative at TOC (mean time to TOC 15 days (range: 7-42). In univariate analysis, only black ethnicity was associated with likelihood of TV BDQ+ (RR 10.2 [95\%CI 2.15-48.4]).

Discussion The use of the BDQ enhanced detection of TV in asymptomatic and symptomatic populations. Cost effective implementation of the test will rely upon further work to reliably detect demographic and clinical variables that predict positivity.

\section{P198 COMPARISON OF RECREATIONAL DRUG USE (RDU) AND SEXUALLY TRANSMITTED INFECTIONS (STIS) IN HIV POSITIVE MEN WHO HAVE SEX WITH MEN (MSM) AND HIV NEGATIVE MSM RECEIVING POST EXPOSURE PROPHYLAXIS (PEP)}

Paul Davis, Vicki Kirby, Andy Rosiak, Brian Thornton, Selena Singh*. Bart's Health NHS Trust, London, UK

\subsection{6/sextrans-2016-052718.246}

Introduction Recreational drug use (RDU) in a sexualised context ("chemsex") is increasing amongst MSM regardless of HIV status and is associated with poor sexual health outcomes. National guidelines for both PEP and HIV management suggest regular sexual health screens (SHS) and screening for RDU and alcohol use.

Aim(s)/objectives To audit the documentation of SHS and RDU history from HIV positive MSM attending routine outpatient clinics compared to HIV negative MSM accessing PEP. 
Methods Retrospective review of 45 randomly selected MSM attending routine HIV care (HIV+ group) OR receiving PEP (PEP group) at each of 2 London clinics during 2014/15.

Results

\begin{tabular}{lll}
\multicolumn{2}{c}{ Abstract P198 Table 1 } & RDU use in HIV+ and HIV negative MSM \\
\hline HIV+ group $(\boldsymbol{n}=\mathbf{9 0})$ & PEP group $(\boldsymbol{n}=\mathbf{8 1})$ \\
\hline Aged $<45$ & $29(32 \%)$ & $67(83 \%)$ \\
Offered SHS & $47(52 \%)$ & $73(90 \%)$ \\
Accepted SHS & $33(70 \%)$ & $68(93 \%)$ \\
Positive for STI & $8(24 \%)$ & $25(37 \%)$ \\
Asked about RDU & $40(44 \%)$ & $78(96 \%)$ \\
Ever used RDU & $18(45 \%)$ & $38(48 \%)$ \\
Used RDU in last 6 months & $8(44 \%)$ & $37(97 \%)$ \\
RDU considered problematic & $2(25 \%)$ & $14(37 \%)$ \\
\hline
\end{tabular}

The most commonly used drug was mephedrone ( $81 \%$ in PEP group vs $38 \%$ in HIV+ group) followed by crystal methamphetamine ( $54 \%$ vs $12.5 \%$ ). $2 / 81(2.4 \%)$ in the PEP group tested HIV positive within 3 months of follow up.

Discussion High levels of STIs and RDU were seen in both groups but most significantly in the PEP group. This highlights the importance of identifying RDU/chemsex in PEP patients, which may be an opportunity for intervention to reduce risk of acquisition of HIV through risky sex.

\section{P199 ANALYSIS OF TRAINING NEEDS IN A NEWLY INTEGRATED SEXUAL HEALTH SERVICE (SHS)}

Belinda Loftus*, Susan Dymock. Spectrum Community Health CIC, Yorkshire, UK

\subsection{6/sextrans-2016-052718.247}

Background The Integrated Sexual Health Services: National Service Specification 2013, includes; Patients to receive their care in a "one stop shop." Staff training should include accredited courses facilitated by BASHH and the FRSH. Key Performance Indicators that relate to the number of staff who are dual qualified.

Method Nurses were identified by their nursing role, band, original speciality and their training needs. The Band 6 (B6) nurses are the initial first training focus. Training for B6 nurses was considered as Essential; (that required to meet service specification) and Non Essential (that required in order to deliver a truly "one stop" holistic care package, or provide training to others).

Aim To identify the training needs of a newly integrated service in order to formulate a strategy which meets the training requirements of the team, whilst retaining high quality service delivery.

Conclusion Integration causes a significant training burden on SHS from a financial, organisational and workforce perspective. Meeting integration training demands is likely to cause disruption to services and staff; resulting in additional stress, increasing sickness and staff turnover rates. Training approach is based upon "quick wins" first targeting those B6 nurses with contraception and Level 2 sexual health experience. B6 Nurses were targeted first as most of their time is spent in clinic; thus improving access, and useful in upskilling others. B6 nurses considered "early adopters" were selected, as likely to have a positive effect on others.

\section{P200 EARLY DETECTION OF SEXUALLY TRANSMITTED INFECTIONS - WERE THERE MISSED OPPORTUNITIES? A QUALITATIVE STUDY IN THE UK}

${ }^{1}$ Hannah Loftus*, ${ }^{2}$ Kelly Mackenzie, ${ }^{3}$ lan Simms, ${ }^{4,3}$ Jackie Cassell. ${ }^{1}$ Sheffield Teaching Hospitals NHS Foundation Trust, Sheffield, UK; ${ }^{2}$ School of Health and Related Research, University of Sheffield, Sheffield, UK; ${ }^{3}$ Public Health England, London, UK; ${ }^{4}$ Division of Primary Care and Public Health, Brighton and Sussex Medical School, Brighton, UK

\subsection{6/sextrans-2016-052718.248}

Background/introduction The early recognition and investigation of outbreaks of sexually transmitted infections (STIs) is vital for preventing onward transmission.

Aim(s)/objectives We sought to understand the facilitators and barriers to outbreak recognition in order to improve early detection. To review the recognition and management of a series of recent outbreaks of sexually transmitted infections in the United Kingdom (UK). To formulate guidance that will enable early recognition of outbreaks.

Methods We interviewed clinicians and public health professionals who had been recently involved in identifying and managing STI outbreaks in the UK. Interviews were audio-recorded and transcribed verbatim. Transcripts were analysed using thematic analysis.

Results Ten STI outbreaks were reviewed, generally by interviewing both a clinician and public health professional. Health advisers and sexual health consultants often noticed increases in cases with smaller clinics often identifying outbreaks more quickly than larger centres through "soft" signals such as increased partner notification, contacts named multiple times or cases with similar geographical location. Sometimes changing demographics first alerted staff. In two centres, increased ceftriaxone use prompted data review. Public Health England (PHE) regional teams identified two outbreaks: one through analysis of the national dataset (GUMCAD); and one via the formal Infectious Diseases Notifications process.

Discussion/conclusion "Soft" signals, picked up in smaller clinics were less readily noticed in larger services. Although quarterly retrospective collation of electronic data by PHE currently limits their role, electronic records should be better exploited locally within services.

\section{P201 EVALUATION OF CHLAMYDIA TRACHOMATIS (CT) AND NEISSERIA GONORRHOEAE (GC) INFECTIONS IN FEMALE SEX WORKERS (FSW) ATTENDING A DEDICATED SEX WORKER CLINIC}

${ }^{1}$ Vanita Devadass, ${ }^{2}$ Kim Leverett, ${ }^{2}$ Sarah Macauley, ${ }^{1}$ Selena Singh*. ${ }^{1}$ Bart's Health NHS Trust, London, UK; ${ }^{2}$ Open Doors (City \& Hackney, Tower Hamlets \& Newham), London, UK

\subsection{6/sextrans-2016-052718.249}

Background/introduction Previous research shows that female sex workers (FSW) are at high risk of certain sexually transmitted infections (STIs), and that migrant FSW appear to be at even higher risk.

Aim(s)/objectives To evaluate the characteristics of FSW managed by our dedicated sex worker clinics who tested positive for either CT or GC, including information about sexual partners outside of work.

Methods Retrospective case note review of patients identified by the Sexual Health and HIV Activity Property Type (SHHAPT) code 'SW' who also had either CT or GC in 2012-2014. 